\title{
DETERMINACIÓN DEL STOCK DE CARBONO EN AGUAJALES DE LA CUENCA DEL RíO AGUAYTÍA, UCAYALI - PERÚ.
}

\author{
Diego GARCIA SORIA ${ }^{1}$, Eurídice HONORIO CORONADO ${ }^{1}$, Dennis DEL CASTILLO
}

1 Instituto de Investigaciones de la Amazonía Peruana, IIAP. Programa en Manejo Integral del Bosque y Servicios Ambientales (PROBOSOUES). Estación Experimental Ucayali. Carretera Federico Basadre Km. 12.400, Pucallpa, Perú. E-mail:dgarcia@iiap.org.pe.

\begin{abstract}
RESUMEN
En el presente estudio se estimó el stock de carbono orgánico del suelo (COS), la biomasa aérea y la necromasa en los aguajales de la parte bajo y alta de la cuenca del río Aguaytía en el departamento de Ucayali, Perú. Para determinar el COS se tomaron muestras de suelo y se determinó el carbono orgánico (\%) y la densidad aparente. El carbono en la biomasa aérea se estimó estableciendo parcelas temporales donde se midió el diámetro a la altura del pecho y altura de todos los árboles y palmeras, y la biomasa se calculó mediante ecuaciones alométricas. La necromasa se estimó midiendo el diámetro de los árboles muertos caídos, el diámetro y la altura de los tocones y árboles muertos en pie, y se pesó la hojarasca en un cuadrante de $1 \mathrm{~m}^{2}$. Los aguajales de la zona baja del Ucayali fueron los que presentaron mayor stock de carbono, en cambio en los aguajales de la zona alta fueron los que almacenaron menos carbono. Los resultados muestran que para los aguajales de la zona alta el COS no está aportando significativamente al stock de carbono, mientras que para la zona baja el COS es el depósito con mayor stock. Así mismo, se pudo observar que los aguajales de la zona alta tienen un mayor grado intervención antrópica, por lo cual, se podrían establecer sistemas de conservación y manejo que permitan incrementar carbono a nivel de paisaje insertando técnicas de cosecha sin tala y conservando otras áreas para dar paso a la regeneración natural de los aguajales.
\end{abstract}

PALABRAS CLAVE: Aguajales, Mauritia flexuosa, carbono, biomasa.

\section{DETERMINATION OF THE CARBON STOCK IN AGUAJALES (AMAZON WET LANDS) OF THE AGUAYTÍA RIVER BASIN, UCAYALI - PERU}

In the present study the soil carbon stock (SCS), the air biomass and the necromass in the aguajales of the lower and higher basins of the Aguaytia Basin in the Peruvian department, Ucayali, were estimated. To determine the SCS, soil measures were taken and the organic carbon (\%) and the apparent density were determined. The carbon of air biomass was estimated by establishing temporary plots were the diameter was measured at chest height and at all the trees and palm's height, and the biomass was calculated with alometric equations. The necromass was estimated by measuring the diameter of the fallen dead trees, the diameter and height of the stumps and the standing dead trees and the weight of the fallen leafs in a $1 \mathrm{~m}^{2}$ quadrant. The aguajales of the Ucayali low zone were the ones that presented major carbon stock, on the other hand, the aguajales of the high zone were those that stored less carbon. The results show that for the aguajales of the high zone the SCS it is not reaching significantly to the carbon stock, whereas for the low zone this one is the warehouse with major carbon stock. Likewise, it was possible to observe that the aguajales from the high zone have a major degree of anthropic intervention, for which, there could be an establishment of conservation and management systems that allow the increase of carbon at a landscape level by inserting technologies of crops without timber and preserving other areas to give step through to the natural regeneration of the aguajales.

KEYWORDS: Aguajales, Mauritia flexuosa, carbon, biomass. 


\section{INTRODUCCIÓN}

En la actualidad los estudios de estimación de carbono en el Perú se realizan principalmente en los bosques de tierra firme, esto ocasiona un vacío de información para las zonas inundables en general y específicamente en áreas con predominancia de aguaje (Mauritia flexuosa) llamadas aguajales, los cuales ocupan un área aproximada de 6'063,551.00 ha, correspondiente al $4.7 \%$ de la superficie nacional (MINAM, 2010). Los pocos estudios que se encuentran en Perú en estos ecosistemas inundados están enfocados en zonas dentro del departamento de Loreto, no reportándose estudios de este tipo en otras regiones.

La relevancia de este estudio es hacer mediciones sobre estimaciones del carbono en la biomasa aérea, necromasa y carbono orgánico del suelo en los aguajales de la cuenca del río Aguaytía del departamento de Ucayali. El departamento de Ucayali tiene 55,781.21 hectáreas de aguajales (GOREU, 2008), localizadas principalmente en las cuencas aluviales (Peter, 2000). Los aguajales son proveedores de beneficios económicos y ambientales importantes para el poblador amazónico (Freitas et al., 2006), así mismo desempeñan un papel importante en la compleja cadena alimenticia del bosque tropical, dado que son hábitats clave para numerosas especies (Bodmer et al., 1997). Además desempeñan una función importante en el ciclo del carbono (IPCC, 1996), pues representan el mayor componente de la acumulación de carbono terrestre (Krankina, 1995, citado por Freitas, 2006) con más de 600 toneladas de carbono por hectárea (IIAP, 2006), por lo que su rol en la mitigación del cambio climático es de gran importancia. De igual modo, estos ecosistemas especiales se presentan en diferentes zonas y condiciones de sitio, con variación en su stock de carbono, por lo cual es importante la medición de los stocks de carbono para hacer la diferenciación entre los diferentes tipos de aguajales. Con esta información se podrá establecer líneas de base más precisas que fortalecerán las políticas ambientales de gobierno

\section{MATERIALES Y MÉTODOS}

\section{ÁREA DEL ESTUDIO}

El presente estudio se realizó en cuatro zonas de la cuenca del río Aguaytía ubicada en la parte norte de la Región Ucayali, Perú (Figura 1). Las zonas 1, 2 y 3 corresponden a los aguajales con altitudes de 150 164 m.s.n.m.; y la zona 4 corresponde a los aguajales con altitudes de 300-360 m.s.n.m. La zona baja forma parte de la zona aluvial inundable influenciado por el río Ucayali (Zona 2), el río
Abujao (Zona 1) y el rio Manantay (Zona 3). Estas zonas se inundan durante 5 meses al año desde noviembre hasta marzo, la precipitación media anual es de $1500 \mathrm{~mm}$, y muestran una topografía de llanura plana. En las 3 zonas mencionadas, las poblaciones locales hacen la cosecha de los frutos de aguaje en forma tradicional, mayormente cortando las palmeras. La zona alta se encuentra influenciada por la quebrada Pintuyacu tributario del río Aguaytía. En esta zona no se presentan inundaciones duraderas, solo por periodos cortos de 48 horas. La precipitación media anual es de $3000 \mathrm{~mm}$, y presenta una topografía ondulada con pendientes moderadamente pronunciadas.

\section{DISEÑO DE LAS PARCELAS DE MUESTREO}

Se establecieron 18 parcelas temporales de 25 x 100 $\mathrm{m}$ para la evaluación de biomasa, necromasa y carbono orgánico del suelo. Diez de estas parcelas están en la zona alta y ocho parcelas en la zona baja, tomando como mínimo una distancia perpendicular $100 \mathrm{~m}$ al curso de agua más secano. En cada parcela se utilizó un diseño de muestreo anidado para la evaluación de los diferentes componentes (Figura 2). La biomasa de árboles con diámetro a la altura del pecho (DAP) igual o mayor a $10 \mathrm{~cm}$ fue medida en la parcela principal $\left(2500 \mathrm{~m}^{2}\right)$, los individuos con DAP de 5 a $9.9 \mathrm{~cm}$ en una parcela de $10 \times 10 \mathrm{~m}$, los individuos con DAP de 1.5 a $4.9 \mathrm{~cm}$ en una parcela de 5 x $5 \mathrm{~m}$ y los individuos de menos de $1.5 \mathrm{~cm}$ de DAP fueron evaluados en pequeños cuadrantes de 1 x $1 \mathrm{~m}$ ubicados aleatoriamente en la parcela principal.

La necromasa de los aguajales se midió en árboles muertos en pie, tocones, árboles caídos y hojarasca. Se utilizaron dos métodos de medición: 1) la evaluación en una línea de $100 \mathrm{~m}$ para madera muerta en el suelo y 2) un transecto de $10 \mathrm{~m}$ x $50 \mathrm{~m}$ para árboles muertos en pie y tocones; en ambos casos incluyendo individuos con DAP $\geq 1.5 \mathrm{~cm}$. La hojarasca se colectó usando las mismas 4 parcelas de $1 \mathrm{~m}^{2}$ descritas anteriormente. Se tomó información adicional sobre las especies.

Para la medición del suelo orgánico (turba), se tomaron muestras a dos profundidades, $0-25 \mathrm{~cm}$ y $25-50 \mathrm{~cm}$ siguiendo el protocolo propuesto por Rugnitz et al. (2009).

\section{BIOMASA AÉREA DEL AGUAJAL}

Para el cálculo de la biomasa aérea en los individuos con DAP mayores a $10 \mathrm{~cm}$ se midió el diámetro a $1.30 \mathrm{~m}$ del suelo utilizando una cinta diamétrica. La estimación de la altura de todos los individuos incluyendo el aguaje se usó un clinómetro según el protocolo estándar de parcelas permanentes de RAINFOR (Phillips \& Baker, 2002). Del mismo 
modo, para el caso de las especies con DAP de 5 a $9.99 \mathrm{~cm}$ y de 1.5 a $4.99 \mathrm{~cm}$ se tomó la altura y el DAP usando una cinta diamétrica y clinómetro. Las ecuaciones alométricas usadas fueron las encontradas en la literatura (Tabla 1) y para los valores de densidad de la madera de cada especie identificada se usó la base de datos disponible en la web (http://hdl.handle.net/10255/dryad.235; Zanne et al., 2009). Así mismo, para el caso de los individuos menores a $1.49 \mathrm{~cm}$ de DAP se instalaron al azar 4 cuadrantes de $1 \mathrm{~m}^{2}$ de donde se colecto la totalidad de la biomasa tomando su peso fresco total (Figura 2). Posteriormente se colecto una muestra de madera para llevar al laboratorio y obtener un peso seco constante a $65^{\circ} \mathrm{C}$, se consideró la fracción de carbono de 0.5 del peso seco obtenido (IPCC, 2003).

\section{CARBONO DE NECROMASA}

Para medir los árboles caídos se usó el método de intersección de línea en el cual se estableció un transecto de $100 \mathrm{~m}$, ubicado en uno de los lados de la parcela (Rugnitz et al., 2009). (Figura 2): En ese transecto se midió los diámetros de los troncos que se interceptan por la línea del transecto, y en el caso de troncos de forma elíptica se tomaron dos diámetros, conjuntamente con la medición del diámetro se clasificó los troncos en tres categorías según su estado de pudrición. Para el cálculo del volumen por hectárea en cada categoría se usó la siguiente ecuación:

Volumen $\left(\mathrm{m}^{3} / \mathrm{ha}\right)=\pi^{2} *\left(\mathrm{D} 1^{2}+\mathrm{D} 2^{2}+\ldots \ldots+\mathrm{Dn}^{2}\right) /(8 * \mathrm{~L})$

Donde:

$\mathrm{D}^{2}+\mathrm{D} 2^{2}+\ldots \ldots+\mathrm{Dn}^{2}=$ Diámetro de cada una de los troncos caídos en centímetros $(\mathrm{cm})$

\section{$\mathrm{L}=$ Longitud de la línea en metros}

Posteriormente para obtener el contenido total de necromasa de las tres categorías se multiplica el resultado de cada categoría por 0.5 para la categoría de árboles sólidos, por 0.3 para la categoría de árboles intermedios y 0.15 para la categoría de árboles podridos.

Para el caso de los árboles muertos en pie y tocones se usó la siguiente formula:

Biomasa $=\pi *\left((\mathrm{D} / 2)^{2} * \mathrm{H}\right) * 0.3 / 1000$

Donde:

$\mathrm{D}=$ Diámetro en $\mathrm{cm}$

$\mathrm{H}=$ Altura en $\mathrm{cm}$.

Para el caso de la necromasa herbácea se instalaron al azar 4 cuadrantes de $1 \mathrm{~m}^{2}$ de donde se colecto la totalidad de la necromasa tomando su peso fresco total, posteriormente se tomó una muestra para llevar al laboratorio en donde se secó a $65^{\circ} \mathrm{C}$ hasta obtener un peso seco constante, se consideró la fracción de carbono de 0.5 del peso seco obtenido (IPCC, 2003) (Figura 2).

\section{CARBONO ORGÁNICO DEL SUELO (COS)}

Para el análisis del carbono orgánico del suelo se consideraron 2 profundidades de muestreo: de 0 a 25 $\mathrm{cm}$ y de 25 a $50 \mathrm{~cm}$. La metodología de cuantificación fue usando la densidad aparente por el método del cilindro. El análisis químico se realizó utilizando el método de Walkley \& Black (1934) en el laboratorio de la Universidad Agraria de la Molina.

\section{RESULTADOS Y DISCUSIÓN}

\section{CARBONOTOTAL}

El carbono orgánico del suelo (COS) de ambas zonas presentó mayor carbono promedio total, sin embargo en el caso de los aguajales de zona alta presento menos carbono total que todos los otros depósitos. En los aguajales de la zona alta el carbono orgánico del suelo $\left(3.78 \pm 1.28 \mathrm{t} \mathrm{ha}^{-1}\right)$ fue significativamente menor en su contenido total de carbono en comparación a los otros depósitos en ambas zonas. Sin embargo, en la zona baja esté mismo depósito mostró significativamente los mayores valores $\left(197.86 \pm 89.32 \mathrm{t} \mathrm{C} \mathrm{ha}^{-1}\right)$ siendo el depósito con mayor carbono total. La biomasa aérea fue significativamente similar en su contenido total de carbono en ambas zonas, de $96.33 \pm 15.16 \mathrm{t} \mathrm{C} \mathrm{ha}^{-1}$ en la zona baja y de $51.28 \pm 16.29 \mathrm{t} \mathrm{C} \mathrm{ha}^{-1}$ en la zona alta (Figura 3 a y b). En los dos tipos de aguajales, la necromasa fue el depósito con menos carbono total.

\section{CARBONO EN LA BIOMASA ÁREA}

En la zona baja se encontró $96.33 \pm 15.16 \mathrm{t} \mathrm{ha}^{-1} \mathrm{de}$ carbono en la biomasa aérea en los aguajales (Figura 4 a). En esta zona, el estrato que presentó mayor contenido de carbono fueron los árboles y palmeras con DAP $\geq 10.00 \mathrm{~cm}$ con un total de $88.68 \mathrm{t} \mathrm{Cha}^{-1}$. El carbono en la biomasa herbácea $\leq 1.5 \mathrm{~cm}$ de DAP mostraron la menor cantidad de carbono almacenado en este estrato $1.10 \mathrm{tC} \mathrm{ha}^{-1}$.

El contenido de carbono en la biomasa aérea de los aguajales reportados en este estudio (entre $51.28 \mathrm{y}$ $96.33 \mathrm{tC} \mathrm{ha}^{-1}$ ) son similares al reporte existente para biomasa aérea de aguajales densos descritos por Freitas et al. (2006) en Pacaya Samiria que reportó (108.81 t C ha $\left.{ }^{-1}\right)$. Sin embargo, los datos no coinciden para la zona alta de la cuenca, en los cuales se reporta un valor más bajo a los reportado en el estudio en Pacaya Samiria (Freitas et al., 2006) que 
reportó $81.29 \mathrm{tC} \mathrm{ha}^{-1}$ en los aguajales mixtos (Figura 4 a). Estas variaciones podría ser atribuida a diferencias en la calidad de sitio de tipo topográfico y de precipitación, así como la mayor exposición de este ecosistema a actividades antrópicas: Es preciso recordar que los aguajales de la zona alta se encuentran cerca a la carreta Federico Basadre la misma que presenta la mayor tasa de deforestación en la amazonía peruana de 30.000 ha por año (Velasquez, 2008).

\section{CARBONO DE NECROMASA}

El nivel de carbono de la necromasa tiene un promedio de $10.41 \mathrm{t} \mathrm{C} \mathrm{ha}^{-1}$ para la zona baja, valor ligeramente mayor a los reportados por Freitas et al., 2006 de $6.59 \mathrm{tC} \mathrm{ha}^{-1}$ en el aguajal denso. El nivel del carbono de la necromasa de la zona alta tiene un promedio de $6.50 \mathrm{t} \mathrm{C} \mathrm{ha}^{-1}$ (Figura $4 \mathrm{~b}$ ), valor ligeramente menor a los reportados por Freitas et al., 2006 que reporta un promedio de $7.38 \mathrm{t} \mathrm{C} \mathrm{ha}^{-1}$ en el aguajal mixto. Estos valores menores encontrados en la zona alta de la cuenca se explican en los resultados del inventario forestal que grafican una diferencia significativa entre los aguajales de bajo y los de altura con una frecuencia absoluta de todas las especies de 563 y 164 individuos/ha, respectivamente. Esto podría deberse a la naturaleza no inundable y a la topografía ondulada de este tipo de aguajal, características que aparentemente no permiten el buen desarrollo del aguaje.

\section{CARBONO ORGÁNICO DEL SUELO (COS)}

Las diferencias encontradas (Figura $4 \mathrm{c}$ ) en los promedios de COS en las parcelas de la zona baja y alta de la cuenca del Aguaytía parecen explicarse por el tipo de topografía del terreno y su precipitación. Según lo observado, en la zona alta de la cuenca se tiene un paisaje con presencia de pendientes y una precipitación promedio de $3000 \mathrm{~mm}$ versus una topografía de llanura y precipitación promedio de $1500 \mathrm{~mm}$ en la zona baja. Las condiciones de la zona alta genera una más rápida perdida de materia orgánica en el suelo al ser ésta lixiviada y arrastrada hacia las quebradas y ríos por la precipitación pluvial, no posibilitando un ambiente anaerobio propicio para retardar la descomposición de la materia orgánica, hecho que si ocurre en la mayor parte aguajales de la zona baja de la cuenca del río Aguaytía.

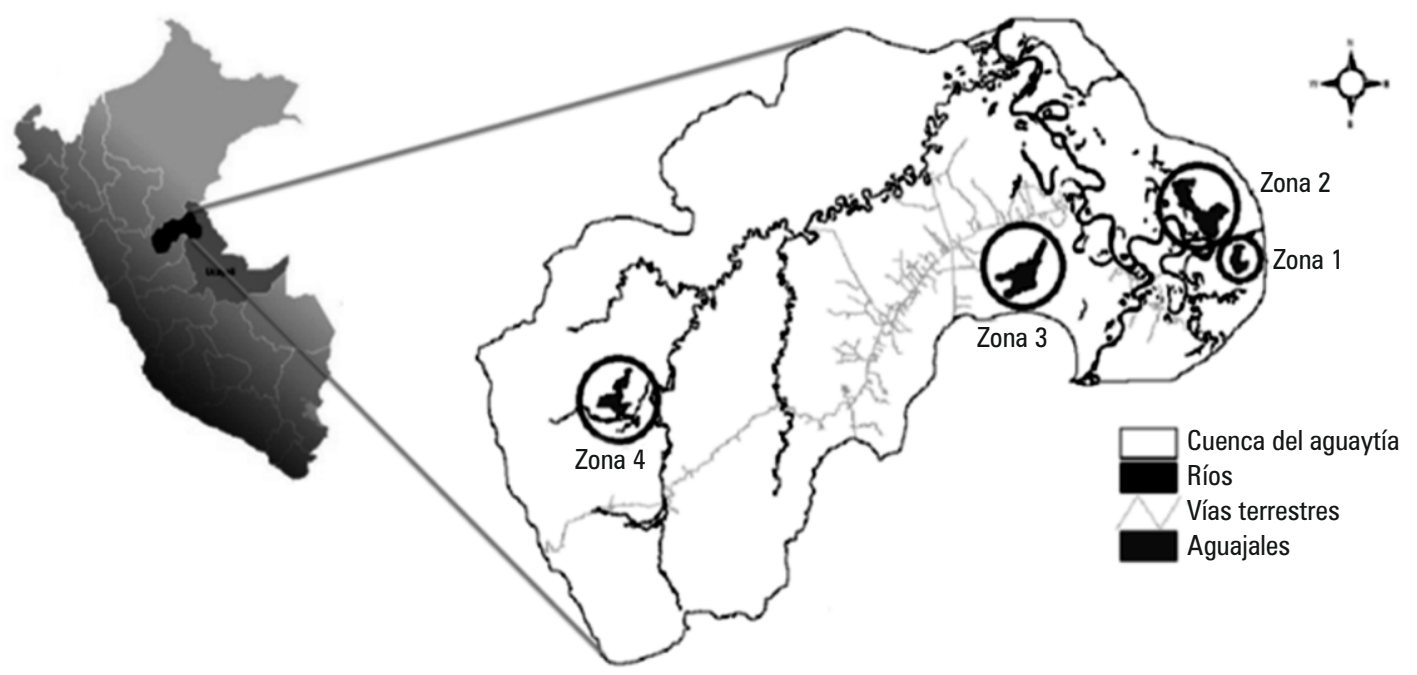

Figura 1. Ubicación de los aguajales en la cuenca del Río Aguaytía, región Ucayali, Amazonía peruana. 


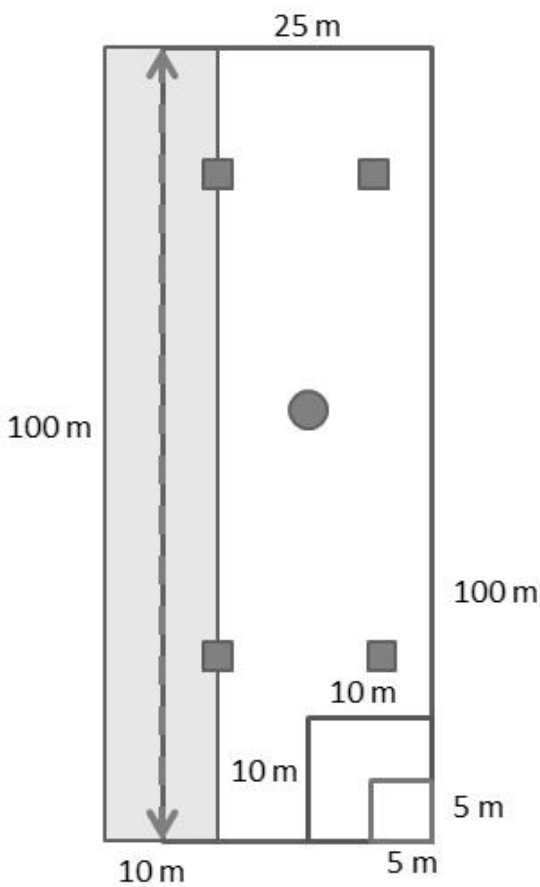

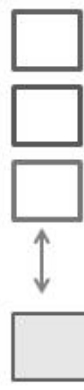

$\mathrm{DAP}>10 \mathrm{~cm}$

DAP 5 a $9.9 \mathrm{~cm}$

DAP 1.5 a $4.9 \mathrm{~cm}$

Árboles caídos

Tocones y arboles muertos en pie

Herbáceas y

hojarasca

Muestra de suelos

en 2 profundidades de 0 a $25 \mathrm{~cm}$ y de 25 a $50 \mathrm{~cm}$.

Figura 2. Diseño anidado de la parcela de muestreo de biomasa, necromasa y de carbono en el suelo. Las medidas de la parcela principal y de las parcelas anidadas se indican en la figura.

Tabla 1. Tamaño de muestra para las ciudades estudiadas.

\begin{tabular}{|c|c|c|}
\hline Especificación & Ecuación & Fuente: \\
\hline \multicolumn{3}{|l|}{ Árboles y otras } \\
\hline palmeras $>10$ & $B=\exp \left(-2.977+\ln \left(p D^{2} H\right)\right)$ & (Chave et al., 2005) \\
\hline \multicolumn{3}{|l|}{$\mathrm{cm}$ de DAP. } \\
\hline \multicolumn{3}{|l|}{ Árboles y otras } \\
\hline palmeras de 5 a & $\mathrm{B}=13.312 * \ln (D A P)-20.237$ & (Chave et al., 2005) \\
\hline \multicolumn{3}{|l|}{$9.9 \mathrm{~cm}$ de DAP. } \\
\hline \multicolumn{3}{|l|}{ Árboles y arbustos } \\
\hline de 1.5 a $4.9 \mathrm{~cm}$ & $B=\exp (-1.7689+2.377 * \ln (D A P))$ & (Nascimento \& Laursnce, 2002) \\
\hline \multicolumn{3}{|l|}{ de DAP. } \\
\hline \multicolumn{3}{|l|}{ Para las } \\
\hline palmeras de & $B=\left(-0.0582^{*} H 3\right)+\left(4.5868^{*} H 2\right)-\left(43.198^{*} H\right)+126.82$ & (Freitas et al., 2006) \\
\hline aguaje. & & \\
\hline
\end{tabular}

Notas: $\mathrm{B}=$ biomasa $(\mathrm{kg}) ; \mathrm{DAP}=$ diámetro a la altura del pecho $(\mathrm{cm}) ; \mathrm{H}=$ altura total $(\mathrm{m})$. 

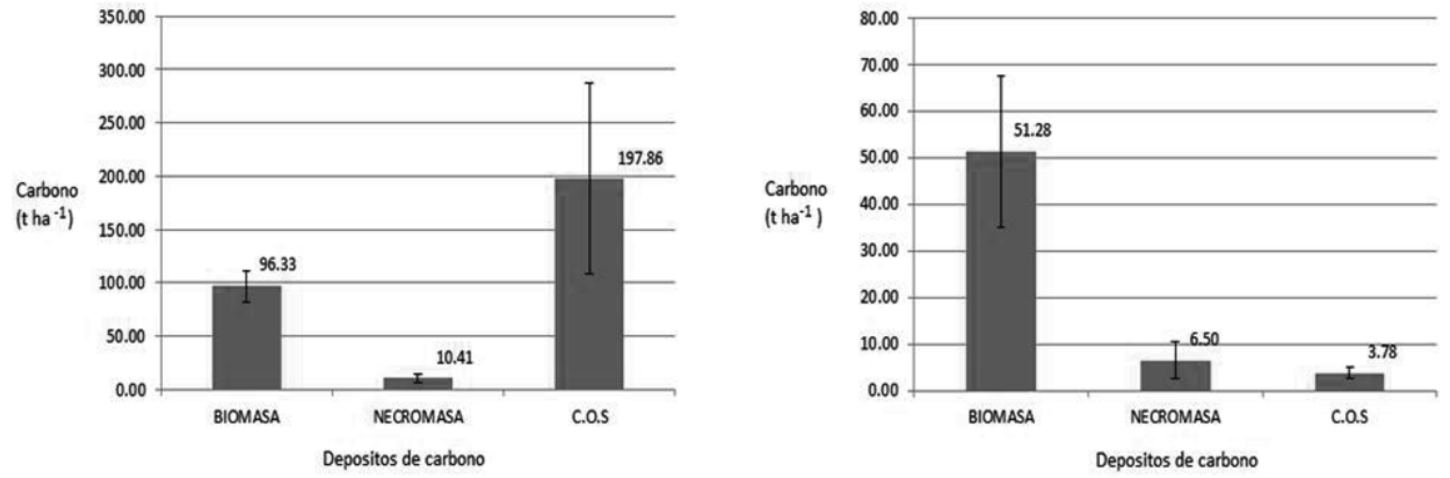

Figura 2. Almacenamiento de carbono total en diferentes depósitos en los aguajales de la cuenca del río Aguaytía (Región Ucayali, Amazonía peruana); a) Agujales de la zona baja y en b) Aguajales de la zona alta. Las barras indican el error estándar.
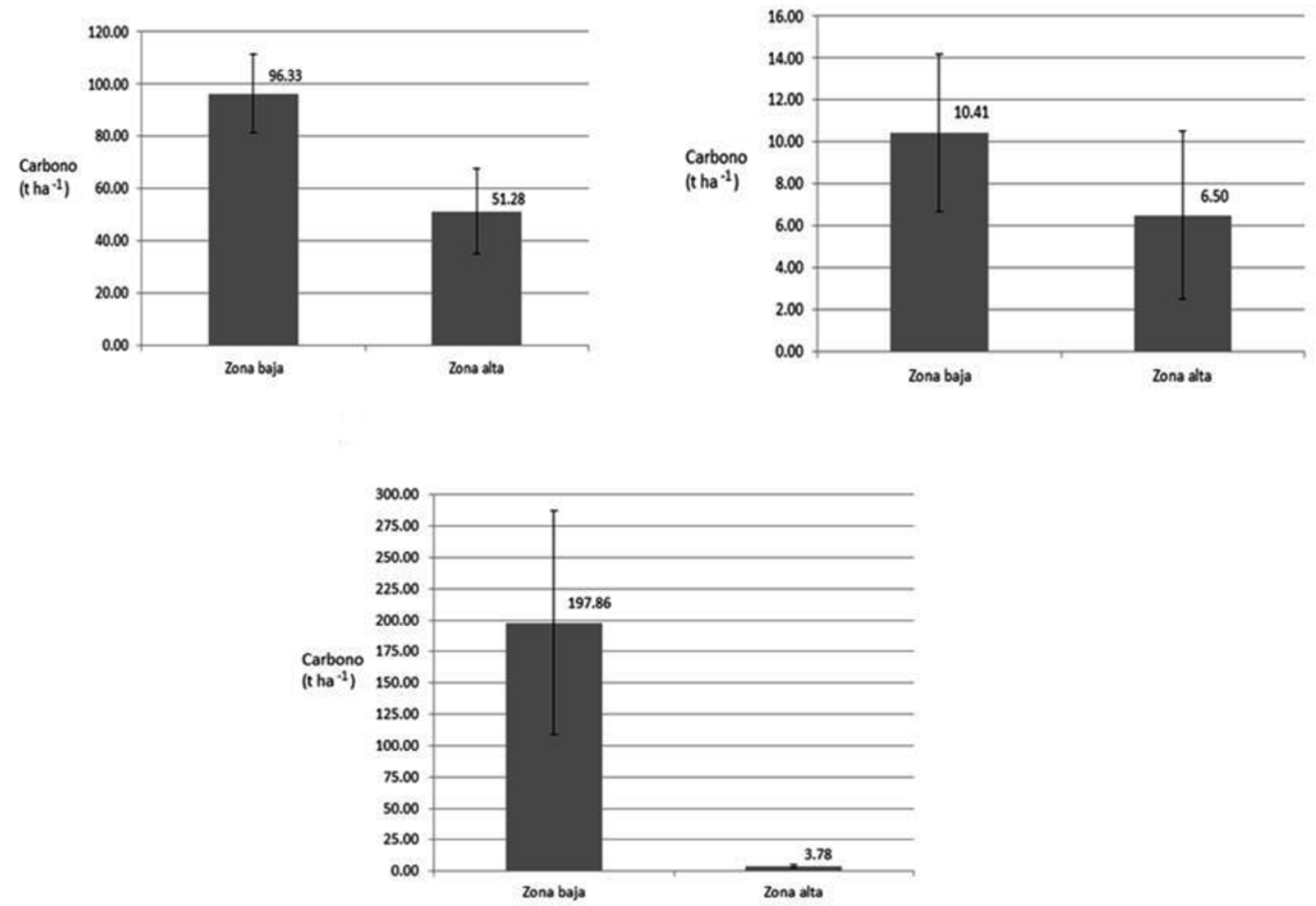

Figura 3. Almacenamiento de carbono total en diferentes depósitos en los aguajales de la cuenca del río Aguaytía (Región Ucayali, Amazonía peruana); a) Agujales de la zona baja y en b) Aguajales de la zona alta. Las barras indican el error estándar. 

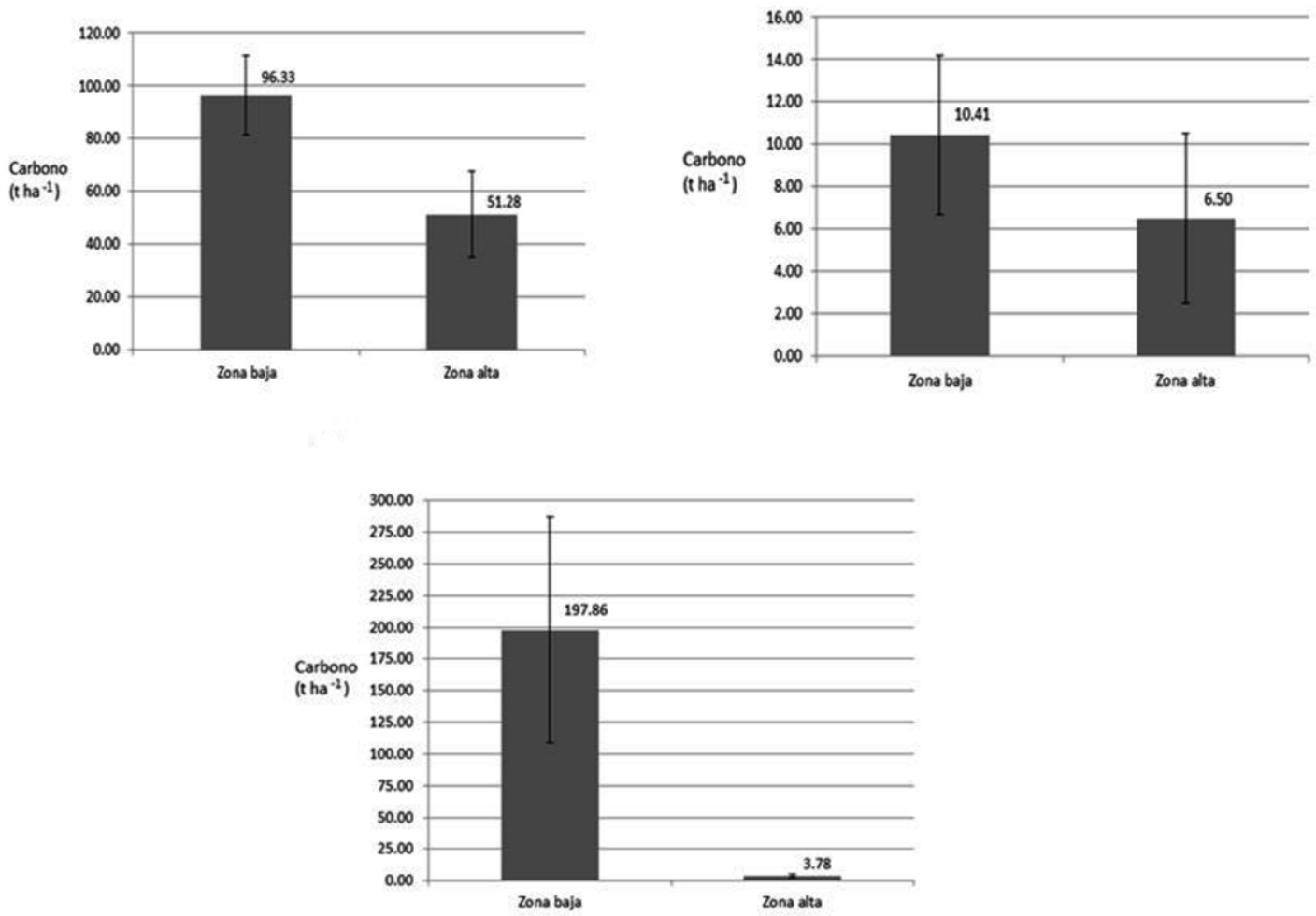

Figura 4. Almacenamiento de carbono por depósito en los aguajales en la zona baja y alta de la cuenca del río Aguaytía (Región Ucayali, Amazonía peruana): biomasa aérea (a), necromasa (b) y $\cos (\mathrm{c})$. 


\section{AGRADECIMIENTO}

Al proyecto REALU de ICRAF y los investigadores, técnicos y asistentes del IIAP, Julio Roca, Rony Ríos y Manuel Rodríguez, por el apoyo constante brindado durante la toma de datos del estudio y todas las evaluaciones.

\section{BIBLIOGRAFÍA CITADA}

Bodmer, R. E.; Aquino, R.; Puertas, P. 1997. Alternativas de manejo para la Reserva Nacional Pacaya Samiria: Un análisis sobre el uso sostenible de la caza. in: T. Fang, R. Bodmer, R. Aquino y M. Valqui (ed.): Manejo de Fauna Silvestre en la Amazonia. Quito, Ecuador, $65-74 \mathrm{p}$.

Honorio-Coronado, E. N.; Baker, T. R. 2010. Manual para el monitoreo del ciclo del carbono en bosques amazónicos. Instituto de Investigaciones de la Amazonia Peruana / Universidad de Leeds. Lima, 54 pp.

Encarnación, F. 1993. El Bosque y las Formaciones Vegetales en la Llanura Amazónica del Perú. Alma Máter. Vol. (6): 95-114.

FAO; CATIE. 1983. Palmeras poco utilizadas de América Tropical. Informe de la reunión de consulta organizada por FAO y CATIE. Turrialba, Costa Rica. 168 pp.

Flores, S. P. 1997. Cultivo de frutales nativos amazónicos. Manual para el extensionista. TCA. Lima. 307pp.

Freitas, L.; Otárola, E.; Del Castillo, D.; Linares, C.; Martínez, P.; Malca, G. 2006. Servicios ambientales de almacenamiento y secuestro de carbono del ecosistema aguajal en la reserva nacional Pacaya Samiria, Loreto - Perú, Documento técnico $\mathrm{N}^{\circ} 29$. Instituto de Investigaciones de la Amazonia Peruana, 65pp

GOREU. 2008. Plan vial departamental participativo de Ucayali 2009 - 2018, Ministerio de Transportes y Telecomunicaciones. Pucallpa. $137 \mathrm{pp}$.

IIAP. 2000. Estudio de las formaciones de palmera en la Reserva Nacional Pacaya-Samiria. Documento Técnico PBIO. 21pp.

IIAP. 2006. Aguaje la Maravillosa Palmera de la Amazonía. Iquitos. 54 pp.

IPCC.1996. Climate Change 1995 - Impacts, Adaptations and mitigation of climate change: scientific technical analysis. Contribution of Working Group II to the Second Assessment Report of the IPCC. Cambridge University Press, Cambridge. 878pp.

IPCC. 2003. Good Practice Guidance for Land Use, Land-Use Change and Forestry, In IPCC Good
Practice Guidance for LULUCF, Chapter 4: Supplementary Methods and Good Practice Guidance Arising from the Kyoto Protocol, Panel on Climate Change, Eds, Jim Penman, Michael Gytarsky, Taka Hiraishi, Thelma Krug, Dina Kruger, Riitta Pipatti, Leandro Buendia, Kyoko Miwa, Todd Ngara, Kiyoto Tanabe and Fabian Wagner. P. 4, 113 - 116.

Malleux, J. 1974. Estudio de la Relación Tipo de Bosque-Especie, en los bosques de la Colonización de Jenaro Herrera. Revista Forestal del Perú. Vol. 5 (1-2) 1-11.

MINAM. 2010. Mapa del Patrimonio Forestall $\mathrm{N} \quad \mathrm{a} \quad \mathrm{c} \quad \mathrm{i} \quad \mathrm{o} \quad \mathrm{n} \quad \mathrm{a} \quad 1$. (http://cdam.minam.gob.pe/novedades/mapap atrimonioforestal.pdf). Acceso: 15/11/2012.

Nascimento, H.; Laurance, W. 2002. Total aboveground biomass in central Amazonian rainforests: a landscape-scale study. Forest Ecology and Management 168, 311-321.

Peter, L.; Nebel, G. 2000. Bosque de la llanura aluvial del Perú: Ecosistemas, habitantes y uso de los recursos. Folia Amazónica. Vol. 10(1-2).

Phillips, O.; Baker, T. 2002. Manual de campo para la remedición y establecimiento de parcelas. Rainfor. Proyecto PAN-AMAZONIA. 18pp.

Rojas, R. 2000. Estado del conocimiento sobre el Aguaje (Mauritia flexuosa L. f.). Iquitos-Perú. 62pp.

Rügnitz, M. T.; Chacón, M. L.; Porro, R. 2009. Guía para la Determinación de Carbono en Pequeñas Propiedades Rurales - 1. ed. - Lima, Perú.: Centro Mundial Agroflorestal (ICRAF) / Consórcio Iniciativa Amazónica(IA). 79 pp.

Velasquez, J. 2008. Los servicios ambientales en la $\mathrm{A} \quad \mathrm{m} \quad \mathrm{a} \quad \mathrm{z} \quad \mathrm{o} \quad \mathrm{n} \quad \mathrm{i} \quad \mathrm{a}$. (http://www.regionucayali.gob.pe/emergentes/ servicios_ambientales).acceso 15/11/2012.

Villachica, $\bar{H}$. 1996. Frutales y Hortalizas promisorios de la Amazonía. Lima-Perú. $367 \mathrm{pp}$.

Walkley, A.; Black, A.I. 1934. An examination of the Degtjoreff method for determination soil organic matter, and a proposed codification of the cromic acid titration method. Soil Science 37:29-38

Zanne, A. E.; López-González, G.; Coomes, D. A.; Ilic, J.; Jansen, S.; Lewis, S. L.; Miller, R. B.; Swenson, N. G.; Wiemann, M. C.; Chave, J. 2009. Global Wood density database. Dryad.

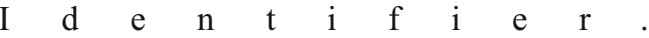
(http://hdl.handle.net/10255/dryad.235.). Acceso: 15/11/2012. 